\title{
IDEAL INTERPOLATION: MOURRAIN'S CONDITION VS. D-INVARIANCE
}

\author{
C. DE BOOR \\ POB 1076, Eastsound, WA 98245, U.S.A. \\ E-mail:deboor@cs.wisc.edu
}

\begin{abstract}
Mourrain [Mo] characterizes those linear projectors on a finite-dimensional polynomial space that can be extended to an ideal projector, i.e., a projector on polynomials whose kernel is an ideal. This is important in the construction of normal form algorithms for a polynomial ideal. Mourrain's characterization requires the polynomial space to be 'connected to 1', a condition that is implied by $D$-invariance in case the polynomial space is spanned by monomials. We give examples to show that, for more general polynomial spaces, $D$-invariance and being 'connected at 1' are unrelated, and that Mourrain's characterization need not hold when his condition is replaced by $D$-invariance.
\end{abstract}

By definition (see [Bi]), ideal interpolation is provided by a linear projector whose kernel is an ideal in the ring $\Pi$ of polynomials (in $d$ real $(\mathbb{F}=\mathbb{R})$ or complex $(\mathbb{F}=\mathbb{C})$ variables). The standard example is Lagrange interpolation; the most general example has been called 'Hermite interpolation' (in [M] and [Bo]) since that is what it reduces to in the univariate case.

Ideal projectors also occur in computer algebra, as the maps that associate a polynomial with its normal form with respect to an ideal; see, e.g., [CLO]. It is in this latter context that Mourrain [Mo] poses and solves the following problem. Among all linear projectors $N$ on

$$
\Pi_{1}(F):=\sum_{j=0}^{d}()_{j} F
$$

with range the linear space $F$, characterize those that are the restriction to $\Pi_{1}(F)$ of an ideal projector with range $F$. Here,

$$
()_{j}:=()^{\varepsilon_{j}}, \quad \varepsilon_{j}:=\left(\delta_{j k}: k=1: d\right), \quad j=0: d,
$$

2000 Mathematics Subject Classification: Primary 41A05, 41A10, 41A63; Secondary 13P10. The paper is in final form and no version of it will be published elsewhere. 
with

$$
()^{\alpha}: \mathbb{F}^{d} \rightarrow \mathbb{F}: x \mapsto x^{\alpha}:=\prod_{j=1}^{d} x(j)^{\alpha(j)}
$$

a handy if nonstandard notation for the monomial with exponent $\alpha$, with

$$
\alpha \in \mathbb{Z}_{+}^{d}:=\left\{\alpha \in \mathbb{Z}^{d}: \alpha(j) \geq 0, j=1: d\right\} .
$$

I also use the corresponding notation

$$
D_{j}
$$

for the derivative with respect to the $j$ th argument, and

$$
D^{\alpha}:=\prod_{j=1}^{d} D_{j}^{\alpha(j)}, \quad \alpha \in \mathbb{Z}_{+}^{d} .
$$

To state Mourrain's result, I also need the following, standard, notations. The (total) degree of the polynomial $p \neq 0$ is the nonnegative integer

$$
\operatorname{deg} p:=\max \{|\alpha|: \widehat{p}(\alpha) \neq 0\},
$$

with

$$
p=: \sum_{\alpha}()^{\alpha} \widehat{p}(\alpha)
$$

and

$$
|\alpha|:=\sum_{j} \alpha(j)
$$

while

$$
\Pi_{<n}:=\{p \in \Pi: \operatorname{deg} p<n\} .
$$

Theorem 1 ([Mo]). Let $F$ be a finite-dimensional linear subspace of $\Pi$ satisfying Mourrain's condition:

$$
f \in F \quad \Longrightarrow \quad f \in \Pi_{1}\left(F \cap \Pi_{<\operatorname{deg} f}\right),
$$

and let $N$ be a linear projector on $\Pi_{1}(F)$ with range $F$. Then, the following are equivalent:

(a) $N$ is the restriction to $\Pi_{1}(F)$ of an ideal projector with range $F$.

(b) The linear maps $M_{j}: F \rightarrow F: f \mapsto N\left(()_{j} f\right), j=1: d$, commute.

For a second proof of this theorem and some unexpected use of it in the setting of ideal interpolation, see [Bo].

Mourrain's condition (2) implies that, if $F$ contains an element of degree $k$, it must also contain an element of degree $k-1$. In particular, if $F$ is nontrivial, then it must contain a constant polynomial. This explains why Mourrain [Mo] calls a linear subspace satisfying his condition connected to 1 . Since the same argument can be made in case $F$ is $D$-invariant, i.e., closed under differentiation, this raises the question what connection if any there might be between these two properties.

In particular, for the special case $d=1$, if $F$ is a linear subspace of dimension $n$ and either satisfying Mourrain's condition or being $D$-invariant, then, necessarily, $F=\Pi_{<n}$. 
More generally, if $F$ is an $n$-dimensional subspace in the subring generated by the linear polynomial

$$
\langle\cdot, y\rangle: \mathbb{F}^{d} \rightarrow \mathbb{F}: x \mapsto\langle x, y\rangle:=\sum_{j=1}^{d} x(j) y(j)
$$

for some $y \neq 0$, then, either way,

$$
F=\operatorname{ran}\left[\langle\cdot, y\rangle^{j-1}: j=1: n\right]:=\left\{\sum_{j=1}^{n}\langle\cdot, y\rangle^{j-1} a(j): a \in \mathbb{F}^{n}\right\} .
$$

As a next example, assume that $F$ is a monomial space (meaning that it is spanned by monomials). If such $F$ is $D$-invariant, then, with each ()$^{\alpha}$ for which $\alpha-\varepsilon_{j} \in \mathbb{Z}_{+}^{d}$, it also contains ()$^{\alpha-\varepsilon_{j}}$ and therefore evidently satisfies Mourrain's condition.

Slightly more generally, assume that $F$ is dilation-invariant, meaning that it contains $f(h \cdot)$ for every $h>0$ if it contains $f$ or, equivalently, $F$ is spanned by homogeneous polynomials. Then every $f \in F$ is of the form

$$
f=: f_{\uparrow}+f_{<\operatorname{deg} f}
$$

with $f_{\uparrow}$ the leading term of $f$, i.e., the unique homogeneous polynomial for which

$$
\operatorname{deg}\left(f-f_{\uparrow}\right)<\operatorname{deg} f
$$

hence in $F$ by dilation-invariance, therefore also

$$
f_{<\operatorname{deg} f} \in F_{<\operatorname{deg} f}:=F \cap \Pi_{<\operatorname{deg} f},
$$

while, by the homogeneity of $f_{\uparrow}$,

$$
\sum_{j=1}^{d}()_{j} D_{j}\left(f_{\uparrow}\right)=(\operatorname{deg} f) f_{\uparrow}
$$

(this is Euler's theorem for homogeneous functions; see, e.g., [Enc: p281] which gives the reference [E: $\S 225$ on p154]). If now $F$ is also $D$-invariant, then $D_{j}\left(f_{\uparrow}\right) \in$ $F_{<\operatorname{deg} f}$, hence, altogether,

$$
f \in \Pi_{1}\left(F_{<\operatorname{deg} f}\right), \quad f \in F .
$$

In other words, if a dilation-invariant finite-dimensional subspace $F$ of $\Pi$ is D-invariant, then it also satisfies Mourrain's condition.

On the other hand, the linear space

$$
\operatorname{ran}\left[()^{0},()^{1,0},()^{1,1}\right]=\left\{()^{0} a+()^{1,0} b+()^{1,1} c: a, b, c \in \mathbb{F}\right\}
$$

fails to be $D$-invariant even though it satisfies Mourrain's condition and is monomial, hence dilation-invariant.

The final example, of a space that is D-invariant but does not satisfy Mourrain's condition, is slightly more complicated. In its discussion, I find it convenient to refer to

$$
\operatorname{supp} \widehat{p}
$$

as the 'support' of the polynomial $p=\sum_{\alpha}()^{\alpha} \widehat{p}(\alpha)$, with the quotation marks indicating that it is not actually the support of $p$ but, rather, the support of its coefficient sequence, $\widehat{p}$. 
The example is provided by the $D$-invariant space $F$ generated by the polynomial

$$
p=()^{1,7}+()^{3,3}+()^{5,0},
$$

hence the 'support' of $p$ is

$$
\operatorname{supp} \widehat{p}=\{(1,7),(3,3),(5,0)\}
$$

(see (4) below). Here are a first few elements of $F$ :

$$
D_{1} p=()^{0,7}+3()^{2,3}+5()^{4,0}, \quad D_{2} p=7()^{1,6}+3()^{3,2},
$$

hence

$$
D_{1} D_{2} p=7()^{0,6}+9()^{2,2}, \quad D_{2}^{2} p=42()^{1,5}+6()^{3,1},
$$

also

$$
D_{1}^{2} p=6()^{1,3}+20^{3,0}, \quad D_{1} D_{2}^{2} p=42()^{0,5}+18()^{2,1},
$$

etc. This shows (see (4) below) that any $q \in \Pi_{1}\left(F_{<\operatorname{deg} p}\right)$ having some 'support' in supp $\widehat{p}$ is necessarily a weighted sum of ()$_{1} D_{1} p$ and ()$_{2} D_{2} p$ (and, perhaps, others not having any 'support' in supp $\widehat{p})$, yet $\left(p,()_{1} D_{1} p,()_{2} D_{2} p\right)$ is linearly independent 'on' supp $\widehat{p}$, as the matrix

$$
\left[\begin{array}{lll}
1 & 1 & 7 \\
1 & 3 & 3 \\
1 & 5 & 0
\end{array}\right]
$$

(of their coefficients indexed by $\alpha \in \operatorname{supp} \widehat{p}$ ) is evidently $1-1$. Consequently,

$$
p \notin \Pi_{1}\left(F_{<\operatorname{deg} p}\right),
$$

i.e., this $F$ does not satisfy Mourrain's condition (as also follows from Proposition 3 below, in view of Theorem 1).

This space also provides the proof that, in Theorem 1, one may not, in general, replace Mourrain's condition by $D$-invariance.

Proposition 3. Let $F$ be the D-invariant space spanned by

$$
p=()^{1,7}+()^{3,3}+()^{5,0} .
$$

Then there exists a linear projector, $N$, on $\Pi_{1}(F)$ with range $F$ for which (b) but not (a) of Theorem 1 is satisfied.

Proof. For $\alpha, \beta \in \mathbb{Z}_{+}^{d}$, set

$$
[\alpha \ldots \beta]:=\left\{\gamma \in \mathbb{Z}_{+}^{d}: \alpha \leq \gamma \leq \beta\right\},
$$

with

$$
\alpha \leq \gamma:=\alpha(j) \leq \gamma(j), j=1: d .
$$

With this, we determine a basis for $F$ as follows.

Since $D^{0,4} p$ is a positive scalar multiple of ()$^{1,3}$, we know, by the $D$-invariance of $F$, that

$$
\left\{()^{\zeta}: \zeta \in[(0,0) \ldots(1,3)]\right\} \subset F .
$$

This implies, considering $D^{2,0} p$, that ()$^{3,0}$, hence also ()$^{2,0}$, is in $F$. Hence, altogether,

$$
F=\Pi_{\Xi_{0}} \oplus \operatorname{ran}\left[D^{\alpha} p: \alpha \in[(0,0) \ldots(1,3)]\right],
$$


with

$$
\Pi_{\Gamma}:=\operatorname{ran}\left[()^{\gamma}: \gamma \in \Gamma\right]
$$

and

$$
\Xi_{0}:=[(0,0) \ldots(1,3)] \cup\{(2,0),(3,0)\} .
$$

This provides the convenient basis

$$
b_{\Xi}:=\left[b_{\xi}: \xi \in \Xi\right]
$$

for $F$, indexed by

$$
\Xi:=\Xi_{0} \cup \Xi_{1}, \quad \Xi_{1}:=[(0,4) \ldots(1,7)],
$$

namely

$$
b_{\xi}:= \begin{cases}()^{\xi}, & \xi \in \Xi_{0} \\ D^{(1,7)-\xi} p, & \xi \in \Xi_{1} .\end{cases}
$$

The following schema indicates the sets $\operatorname{supp} \widehat{p}, \Xi_{0}$, and $\Xi_{1}$, as well as the sets $\partial \Xi_{0}$ and $\partial \Xi_{1}$ defined below:

$$
\begin{array}{cccccl}
\times & \times & & & \otimes: & \operatorname{supp} \widehat{p} \\
1 & 1 \otimes & \times & & 0: & \Xi_{0} \\
1 & 1 & \times & & 1: & \Xi_{1} \\
1 & 1 & \times & & +: & \partial \Xi_{0} \\
1 & 1 & \times & & & \\
0 & 0 & + & \otimes & & \\
0 & 0 & + & & & \\
0 & 0 & + & + & & \\
0 & 0 & 0 & 0 & +\otimes &
\end{array}
$$

Now, let $N$ be the linear projector on $\Pi_{1}(F)$ with range $F$ and kernel $\operatorname{ran}\left[b_{\mathrm{Z}}\right]$, with $b_{\mathrm{Z}}$ obtained by thinning

$$
\left[b_{\Xi},()_{1} b_{\Xi},()_{2} b_{\Xi}\right]
$$

to a basis $\left[b_{\Xi}, b_{\mathrm{Z}}\right]$ for $\Pi_{1}(F)$. This keeps the maps $M_{j}: F \rightarrow F: f \mapsto N\left(()_{j} f\right)$ very simple since, as we shall see, for many of the $\xi \in \Xi,()_{j} b_{\xi}$ is an element of the extended basis $\left[b_{\Xi}, b_{\mathrm{Z}}\right]$, hence $N$ either reproduces it or annihilates it.

Specifically, it is evident that the following are in $F$, hence not part of $b_{Z}$ :

$$
\begin{array}{ll}
()_{1} b_{\xi}, & \xi \in[(0,0) \ldots(0,2)], \\
()_{2} b_{\xi}, & \xi \in[(0,0) \ldots(1,3)],
\end{array}
$$

with ()$_{2} b_{\xi} \in F$ for $\xi=(0,3),(1,3)$ since $D^{(1,6)-\xi} p$ and ()$^{\xi+(2,-3)}$ are in $F$. Further, for each

$$
\zeta \in \partial \Xi_{0} \cup \partial \Xi_{1}
$$

with

$$
\partial \Xi_{0}:=\{(2,3),(2,2),(2,1),(3,1),(4,0)\}, \quad \partial \Xi_{1}:=\{[(2,4) \ldots(2,7)],(1,8),(0,8)\},
$$

there is $\xi \in \Xi$ so that, for some $j, \zeta-\xi=\varepsilon_{j}$. Set, correspondingly,

$$
b_{\zeta}:=()_{j} b_{\xi}
$$


Then, none of these is in $F$, and, among them, each $b_{\zeta}$ is the only one having some 'support' at $\zeta$, hence they form a linearly independent sequence. Therefore, each such $b_{\zeta}$ is in $b_{\mathrm{Z}}$.

The remaining candidates for membership in $b_{\mathrm{Z}}$ require a more detailed analysis. We start from the 'top', showing also along the way that (b) of Theorem 1 holds for this $F$ and $N$ by verifying that

$$
M_{1} M_{2}=M_{2} M_{1} \text { on } b_{\xi}
$$

for every $\xi \in \Xi$.

$\xi=(1,7)$ : As already pointed out, both ()$_{1} b_{1,7}$ and ()$_{2} b_{1,7}$ are in $b_{\mathrm{Z}}$, hence (5) holds trivially for $\xi=(1,7)$.

$\xi=(0,7),(1,6)$ : Both ()$_{1} b_{0,7}=()^{1,7}+3()^{3,3}+5()^{5,0}$ and ()$_{2} b_{1,6}=7()^{1,7}+3()^{3,3}$ have their 'support' in that of $p=b_{1,7}=()^{1,7}+()^{3,3}+()^{5,0}$, while, as pointed out and used earlier, the three are independent. Hence ()$_{1} b_{0,7},()_{2} b_{1,6} \in b_{\mathrm{Z}}$, while we already pointed out that ()$_{2} b_{0,7},()_{1} b_{1,6} \in b_{\mathrm{Z}}$, therefore (5) holds trivially.

$$
\xi=(0,6),(1,5) \text { : Both }()_{1} b_{0,6}=7()^{1,6}+9()^{3,2} \text { and }()_{2} b_{1,5}=42()^{1,6}+6()^{3,2} \text { have }
$$
their 'support' in that of $b_{1,6}=7()^{1,6}+3()^{3,2}$, but neither is a scalar multiple of $b_{1,6}$. Hence, one is in $b_{Z}$ and the other is not. Which is which depends on the ordering of the columns of $\left[b_{\Xi},()_{1} b_{\Xi},()_{2} b_{\Xi}\right]$. Assume the ordering such that ()$_{2} b_{1,5} \in b_{\mathrm{Z}}$. Then, since we already know that ()$_{1} b_{1,5} \in b_{\mathrm{Z}}$, (5) holds trivially for $\xi=(1,5)$. Further, ()$_{1} b_{0,6}=4 b_{1,6}-(1 / 2)()_{2} b_{1,5}$, hence $M_{1} b_{0,6}=4 b_{1,6}$, while we already know that ()$_{2} b_{1,6} \in b_{\mathrm{Z}}$ therefore, $M_{2} M_{1} b_{0,6}=0$. On the other hand, ()$_{2} b_{0,6}=7()^{0,7}+3()^{3,3}$ has its 'support' in that of $b_{0,7}=()^{0,7}+3()^{3,3}+5()^{4,0}$ but is not a scalar multiple of it, hence is in $b_{\mathrm{Z}}$, and therefore already $M_{2} b_{0,6}=0$. Thus, (5) also holds for $\xi=(0,6)$.

$\xi=(0,5),(1,4):$ Both ()$_{1} b_{0,5}=42()^{1,5}+18()^{3,1}$ and ()$_{2} b_{1,4}=210()^{1,5}+6()^{3,1}$ have their 'support' in that of $b_{1,5}=42()^{1,5}+6()^{3,1}$ but ()$^{3,1}=b_{3,1}$ was already identified as an element of $b_{\mathrm{Z}}$, hence neither ()$_{1} b_{0,5}$ nor ()$_{2} b_{1,4}$ is in $b_{\mathrm{Z}}$. But, since ()$^{3,1} \in b_{\mathrm{Z}}$, and so $b_{1,5}=N b_{1,5}=N\left(42()^{1,5}\right)$, we have $M_{1} b_{0,5}=b_{1,5}$ and $M_{2} b_{1,4}=5 b_{1,5}$. Since we already know that ()$_{1} b_{1,5} \in b_{\mathrm{Z}}$, it follows that $M_{1} M_{2} b_{1,4}=0$ while we already know that ()$_{1} b_{1,4} \in b_{\mathrm{Z}}$, hence already $M_{1} b_{1,4}=0$. Therefore, (5) holds for $\xi=(1,4)$. Further, we already know that ()$_{2} b_{1,5} \in b_{\mathrm{Z}}$, hence $M_{2} M_{1} b_{0,5}=0$, while ()$_{2} b_{0,5}=$ 42()$^{0,6}+18()^{2,2}$ has the same 'support' as $b_{0,6}=7()^{0,6}+9()^{2,2}$ but is not a scalar multiple of it, hence is in $b_{\mathrm{Z}}$ and, therefore, already $M_{2} b_{0,5}=0$, showing that (5) holds for $\xi=(0,5)$.

$\xi=(0,4):()_{2} b_{0,4}=210()^{0,5}+18()^{2,1}=5 b_{0,5}-72 b_{2,1}$, with $b_{2,1} \in b_{\mathrm{Z}}$, hence ()$_{2} b_{0,4}$ is not in $b_{\mathrm{Z}}$ and $M_{2} b_{0,4}=5 b_{0,5}$, therefore $M_{1} M_{2} b_{0,4}=5 M_{1} b_{0,5}=5 b_{1,5}$, the last equation from the preceding paragraph. On the other hand, ()$_{1} b_{0,4}=210()^{1,4}+18()^{3,0}=b_{1,4}+$ $12 b_{3,0}$, with both $b_{1,4}$ and $b_{3,0}$ in $F$, hence ()$_{1} b_{0,4}$ is not in $b_{\mathrm{Z}}$, and $M_{1} b_{0,4}=b_{1,4}+12 b_{3,0}$, therefore, since ()$_{2} b_{3,0}=b_{3,1} \in b_{\mathrm{Z}}, M_{2} M_{1} b_{0,4}=M_{2} b_{1,4}=5 b_{1,5}$, the last equation from the preceding paragraph. Thus, (5) holds for $\xi=(0,4)$.

$\xi=(1,3)$ : We already know that ()$_{1} b_{1,3}=b_{2,3} \in b_{\mathrm{Z}}$ and therefore already $M_{1} b_{1,3}=0$, while ()$_{2} b_{1,3}=()^{1,4}=\left(b_{1,4}-6 b_{3,0}\right) / 210 \in F$, therefore $210 M_{1} M_{2} b_{1,3}=M_{1} b_{1,4}=0$, thus (5) holds for $\xi=(1,3)$. 
For the remaining $\xi \in \Xi$, each $b_{\xi}$ is a monomial, hence ()$_{j} b_{\xi}$ is again a monomial, and either in $F$ or not and, if not, then its exponent is in

$$
\partial \Xi_{0}:=\{(2,3),(2,2),(2,1),(3,1),(4,0)\} .
$$

Moreover, ()$_{1}()_{2} b_{\xi}$ is in $F$ iff ()$_{2}()_{1} b_{\xi}$ is. Hence, (5) also holds for the remaining $\xi \in \Xi$. This finishes the proof that, for this $F$ and $N$, (b) of Theorem 1 holds.

It remains to show that, nevertheless, (a) of Theorem 1 does not hold. For this, observe that ()$^{2,1}$ and ()$^{4,0}$ are in ker $N$, as is, e.g., ()$_{2} b_{1,6}=7()^{1,7}+3()^{3,3}$, hence $p=$ ()$^{1,7}+()^{3,3}+()^{5,0}$ is in the ideal generated by ker $N$, making it impossible for $N$ to be the restriction to $\Pi_{1}(F)$ of an ideal projector $P$ with range $F$ since this would place the nontrivial $p$ in both $\operatorname{ker} P$ and $\operatorname{ran} P$.

\section{References}

[Bi] G. Birkhoff, The algebra of multivariate interpolation, in: Constructive Approaches to Mathematical Models, C. V. Coffman and G. J. Fix (eds.), Academic Press, New York, 1979, 345-363.

[Bo] C. de Boor, Ideal interpolation, in: Approximation Theory XI: Gatlinburg 2004, C. K. Chui, M. Neamtu, and L. L. Schumaker (eds.), Nashboro Press, Brentwood TN, 2005, 59-91.

[CLO] David Cox, John Little, and Donal O'Shea, Ideals, Varieties, and Algorithms, Undergraduate Texts in Math., Springer-Verlag, New York, 1992.

[Enc] Encyclopädie der mathematischen Wissenschaften, 1. Band, Teubner, Leipzig, 18981904.

[E] L. Euler, Calculus differentialis 1, Ticini, Italy, 1787.

[M] H. M. Möller, Mehrdimensionale Hermite-Interpolation und numerische Integration, Math. Z. 148 (1976), 107-118.

[Mo] B. Mourrain, A new criterion for normal form algorithms, in: Applied Algebra, Algebraic Algorithms and Error-Correcting Codes (Honolulu, Hawaii, 1999), M. Fossorier, H. Imai, S. Lin, A. Pol (eds.), Lecture Notes in Computer Science 1719, Springer-Verlag, Heidelberg, 1999, 430-443. 